\title{
Prevalencia de defectos del desarrollo del esmalte en los incisivos permanentes de escolares: un estudio fotográfico en el Sur de Brasil
}

Prevalence of development defects in permanent incisors enamel in school children:

\section{a photographic study in Southern Brasil}

\author{
Renata Barbieri Bogo ${ }^{1}$ \\ Judith Ángelica Gonzáles Sullcahuamán ${ }^{2}$ \\ Fernanda de Morais Ferreira ${ }^{3}$ \\ José Vitor Nogara Borges de Menezes ${ }^{4}$ \\ Renato Cordeiro Gugisch ${ }^{5}$ \\ Fabian Calixto Fraiz ${ }^{6}$
}

\section{Resumen}

El objetivo de este estudio fue evaluar la prevalencia y distribución de diferentes tipos de problemas de desarrollo del esmalte (DDE) en los incisivos de los escolares de una población de la pequeña ciudad del sur de Brasil. Examinamos a todos los niños y adolescentes que asistieron, del 5 al $8^{\circ}$ grado de educación en las escuelas públicas en la ciudad de Camboriu - SC, cuyas escuelas y cuyos padres aceptaron de forma individual y dieron su consentimiento para su participación. Fueron incluidos en el estudio aquellos que presentaban incisivos con al menos tres cuartas partes de la corona en erupción y en condiciones de ser examinados ( $\sin$ aparatología ortodontica, fractura, lesión cariosa o restauración extensa, $n=223$ ). Dos evaluadores, previamente capacitados utilizaron los criterios clínicos del "FDI World Dental Federation" para el diagnóstico de DDE, a través de proyección de imágenes de fotografías de los incisivos. Análisis descriptivo y test de Chi-cuadrado. 1728 dientes se clasificaron de acuerdo a la pre- sencia de DDE. La prevalencia de DDE fue del $34,1 \%$ para los escolares, siendo que $10,3 \%$ de los dientes examinados presentaron algún tipo de DDE. La prevalencia de las diferentes alteraciones en los incisivos examinados fueron: opacidad difusa $(6,6 \%)$, opacidad demarcada $(3,4 \%)$ e hipoplasia $(0,4 \%)$ y las clasificaciones mas frecuentes fueron la opacidad difusa lineal $(3,7 \%)$, seguido por opacidad blanca demarcada (3\%) y opacidad difusa irregular $(2,8 \%)$. Hubo una asociación estadísticamente significativa $(\mathrm{p}<0,001)$ entre la presencia de DDE y la arcada dentaria, siendo los incisivos superiores más afectados. Los incisivos centrales fueron estadísticamente más afectados $(p<0,001)$ que los laterales. Los incisivos inferiores mostraron una prevalencia similar de DDE entre sí. No hubo diferencias estadísticamente significativas en la prevalencia de DDE en función del género y el lado del diente. Se concluye que la presencia de DDE en los incisivos permanentes de los escolares en el sur de Brasil fue alta, es importante que el cirujano dentista sea capaz de diagnosticar e identificar los posibles factores etiológicos. 
Palabras clave: Esmalte Dental; Anomalias Dentarias; Dentición Permanente; Prevalencia; Hipoplasia del Esmalte Dental.

\section{Abstract}

The main goal of this study was to evaluate the prevalence and distribution of development defects of enamel (DDE) on incisors from schoolchildren from a southern Brazilian city. Schoolchildren attending from 5 th to 8 th grade on public schools from the city of Camboriu, state of Santa Catarina, whose parents agreed to sign the informed consents, participated on the study. Inclusion criteria: children which upper and lower incisors had at least $3 / 4$ of the crown erupted. Exclusion criteria: children under orthodontic treatment, fractured teeth, teeth with carious lesions or any kind of restorative material. 223 schoolchildren were examined. Two examiners, previously calibrated, used the FDI protocol to the DDE exams based on the projection of a photographic image of the upper and lower incisors. The statistical approach was carried out using descriptive analysis and the chi-square test. The results were as follows. The DDE prevalence was $34,1 \%$ and $10,3 \%$ of the teeth examined showed some kind of DDE. The prevalence of the different types of DDE were $6,6 \%$ for diffuse opacities, 3,4\% for demarcated opacities and $0,4 \%$ for hypoplasias. The most frequent classifications were linear diffuse opacities with $3,7 \%$ followed by white demarcated opacities with $3 \%$ and irregular diffuse opacities with $2,8 \%$. A statistical significant difference was found $(p<0,001)$ when analyzing the presence of DDE and the dental arch, with the upper incisors being the most affected. The central incisors were most affected than the lateral ones $(p<0,001)$. No statistical differences were found when analyzing gender or the side of the dental arch. The authors concluded that the prevalence of DDE's on schoolchildren on a southern Brazilian city was high and the dentists should be able to make the correct diagnostic and define possible etiologic factors.

Key words: Dental Enamel; Tooth Abnormalities; Dentition, Permanent; Prevalence; Dental Enamel Hypoplasia.

\section{Introducción}

El esmalte es considerado un marcador biológico, sensible a una serie de eventos y situaciones, y la interpretación de los defectos de desarrollo del esmalte (DDE) puede ser un poderoso instrumento de diagnóstico, tanto de individuos cuenta su ubicación, dientes afectados, extensión, coherencia cronológica y la simetría, aliada a un adecuado levantamiento de informaciones sobre la historia de la salud de un individuo pueden indicar si el defecto se asocia a factores locales o sistémicos y permitir definir la época en que se produjo el evento.

De esta forma, los DDE vienen siendo utilizados como un indicativo del nivel de salud de poblaciones prehispánicas (Griffin, Donlon, 2009) y su asociación con la calidad de vida presenta cada vez mas evidencias científicas. Tanto en los aspectos generales de una determinada población incluyendo los factores socioeconómicos y nutricionales (Massoni, Chaves, Rosenblatt, Oliveira, 2007), como en situaciones especificas como por ejemplo, niños con enfermedad celíaca(Majorana, et al, 2010), asma (Guergolette et al, 2009), tetralogía de Fallot (Assunção, 2008) o histórico de infecciones en la infancia (Arnadóttir, Sigurións, Holbrook, 2005). 
Por otra parte, existe una clara asociación entre traumatismos alveolo dentarios (Da Silva Assunção et al., 2009; do Espíritu Santo Jácomo, Campos, 2009) y las lesiones periapicales en los dientes deciduos con las alteraciones en la formación del esmalte del diente permanente sucesor, es probable que una atención odontológica adecuada en los primeros años de vida disminuya la posibilidad de alteraciones del esmalte en dientes permanentes.

Dentro de los aspectos que alertan la necesidad de estudios en esta área, se destaca la posibilidad de utilizar los índices de DDE como un indicador del nivel de calidad de vida de una determinada población, incluyendo el nivel de atención odontológica. Otro indicador importante de estudiar y monitorizar es la posibilidad de adoptar medidas preventivas, ya que existen pocos factores etiológicos incontrolables asociados a los DDE como es el caso de enfermedades genéticas. Mismo en esta situación, el conocimiento es importante para el correcto diagnóstico y resolución clínica. (Gonzales-Pinedo, Perona-Miguel de Priego, 2009).

Con este trabajo buscamos verificar la prevalencia y distribución de los diferentes tipos de defectos de desarrollo del esmalte en los incisivos de los escolares de una ciudad con poca población del sur de Brasil, lo que permitirá el establecimiento de estrategias de acción encaminadas a la prevención, control y seguimiento.

\section{Material y métodos}

Después de la aprobación por el Comité de Ética del Hospital de Clínicas de la Universidad Federal de Paraná (CEP-HC 052EXT006/2002-04) y la autorización de la prefectura de la ciudad de Camboriú, fue desarrollado en 2002 un estudio transversal con una población constituida por niños y adolescentes, de ambos géneros, matriculados en las escuelas públicas en la ciudad de Camboriú, Santa Catarina, Brasil. Se verificó que el municipio cuenta con el suministro de agua fluorada desde 1983.

Para la selección de los estudiantes, fueron adoptados los siguientes criterios de inclusión: estar matriculados entre el 5to. y el 8vo. grado primario y tener todos los incisivos centrales erupcionados, con exposición por lo menos de tres cuartas partes de la corona. Fueron excluidos los estudiantes de escuelas que no querían participar o cuyos responsables no autorizaron la participación, además de aquellos estudiantes cuyos incisivos no se encontraban en condiciones de ser evaluados (utilizando aparatología ortodontica, con fractura, lesión cariosa extensa o restauración).

De acuerdo con estos criterios, se examinaron 229 niños y adolescentes de tres escuelas públicas. Los exámenes para diagnóstico de DDE fueron a través de fotografías de los incisivos de niños. Antes de ser fotografiados, los escolares recibieron instrucciones de higiene bucal y realizaron cepillado dentario bajo la supervisión directa de los examinadores para el retiro de placa dental. Los cepillos y dentífricos utilizados se donaron a los niños participantes.

Se obtuvieron imágenes de los incisivos con magnificación de 1:1 utilizando máquina fotográfica con lente de $100 \mathrm{~mm}$ y flash circular (Dental EYE® III - Yashica) y rollo Kodak® Ektachrome 100. Las fotografías dentales se realizaron sobre luz natural en el propio patio de las escuelas, por un fotógrafo posicionado en frente de cada niño. Fue utilizado un abridor de boca con el número de identificación visible en el campo fotográfico y el niño fue instruido a mantener una relación en topo para los incisivos. 
Nombre, número y edad del niño se anotaron en una ficha específica.

Después de procesados, los dispositivos fueron codificados y colocados en una secuencia aleatoria, para garantizar una evaluación ciega. Los dispositivos fueron proyectados en una sala oscura sobre tela blanca, usando un equipamiento Kodak ${ }^{\circledR}$ Ektagraphic III y visualizados en una magnificación de 20 veces a una distancia aproximada de 5 metros. Dos examinadores (FCF y JVNBM) previamente entrenados observaron conjuntamente las imágenes y anotaron en ficha propia la condición del esmalte de la cara vestibular de cada uno de los incisivos. En caso de discordancia en cuanto a la clasificación, los conceptos eran revisados con nuevos procedimientos de clasificación, si continuaba la discordancia, era anotado el menor índice.

Los códigos y criterios adoptados fueron los sugeridos por el FDI- World Dental Federation (COMISSION ON ORAL HEALTH, RESEARCH AND EPIDEMIOLOGY-FDI, 1992).

Los datos se sometieron a análisis descriptivos y a la asociación entre la variable respuesta DDE, dicotómica (presente / ausente) o clasifica- das de acuerdo con sus sub-divisiones, y las demás variables independientes fueron evaluadas por el test Chi-cuadrado a través del programa Epi-info versión 3.5.1. El nivel de significancia adoptado fue de $5 \% \quad(a=0,05)$.

\section{Resultados}

De los 229 niños examinados, seis fueron excluidos debido a la imposibilidad de determinación del índice (utilización de aparatología ortodóntica fija), entonces se determinó el resultado en un total de 223 niños con edad media de 12, 06 $( \pm 1,26)$ años.

La prevalencia de DDE en la muestra estudiada fue de $34,1 \%$; es decir que 76 niños presentaban por lo menos un incisivo con alguna alteración de desarrollo del esmalte, sin diferencias entre los géneros (tabla 1).

La tabla 2 presenta las diversas clasificaciones de DDE de acuerdo con los dientes. Fueron fotografiados 1832 dientes, siendo excluidos aquellos que estaban mal posicionados lo que perjudicaba la clasificación o cuya imagen estaba sin foco, resultando en un total de 1728 incisivos evaluados, de los cuales $10,3 \%$ fue diagnosti-

Tabla 1. Distribución de los niños escolares de la red pública de la ciudad de Camboriú, SC, en función de la prevalencia de los defectos de desarrollo del esmalte e del género. 2002, n=223.

\begin{tabular}{|c|c|c|c|c|c|c|c|}
\hline \multirow{3}{*}{$\begin{array}{l}\text { Género } \\
\text { Masculino }\end{array}$} & \multicolumn{6}{|c|}{ Defectos del Desarrollo del Esmalte } & \multirow{2}{*}{ valor de $\mathrm{p}^{*}$} \\
\hline & \multicolumn{2}{|c|}{ Presente } & \multicolumn{2}{|c|}{ Ausente } & \multicolumn{2}{|c|}{ Total } & \\
\hline & 31 & $32,3 \%$ & 65 & $67,7 \%$ & 96 & $100 \%$ & \\
\hline Femenino & 45 & $35,4 \%$ & 82 & $64,6 \%$ & 127 & $100 \%$ & \\
\hline Total & 76 & $34,1 \%$ & 147 & $65,9 \%$ & 223 & $100 \%$ & \\
\hline
\end{tabular}

*Teste Chi-quadrado. 


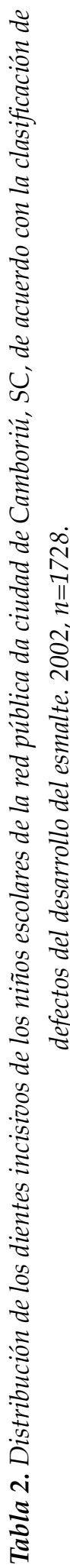

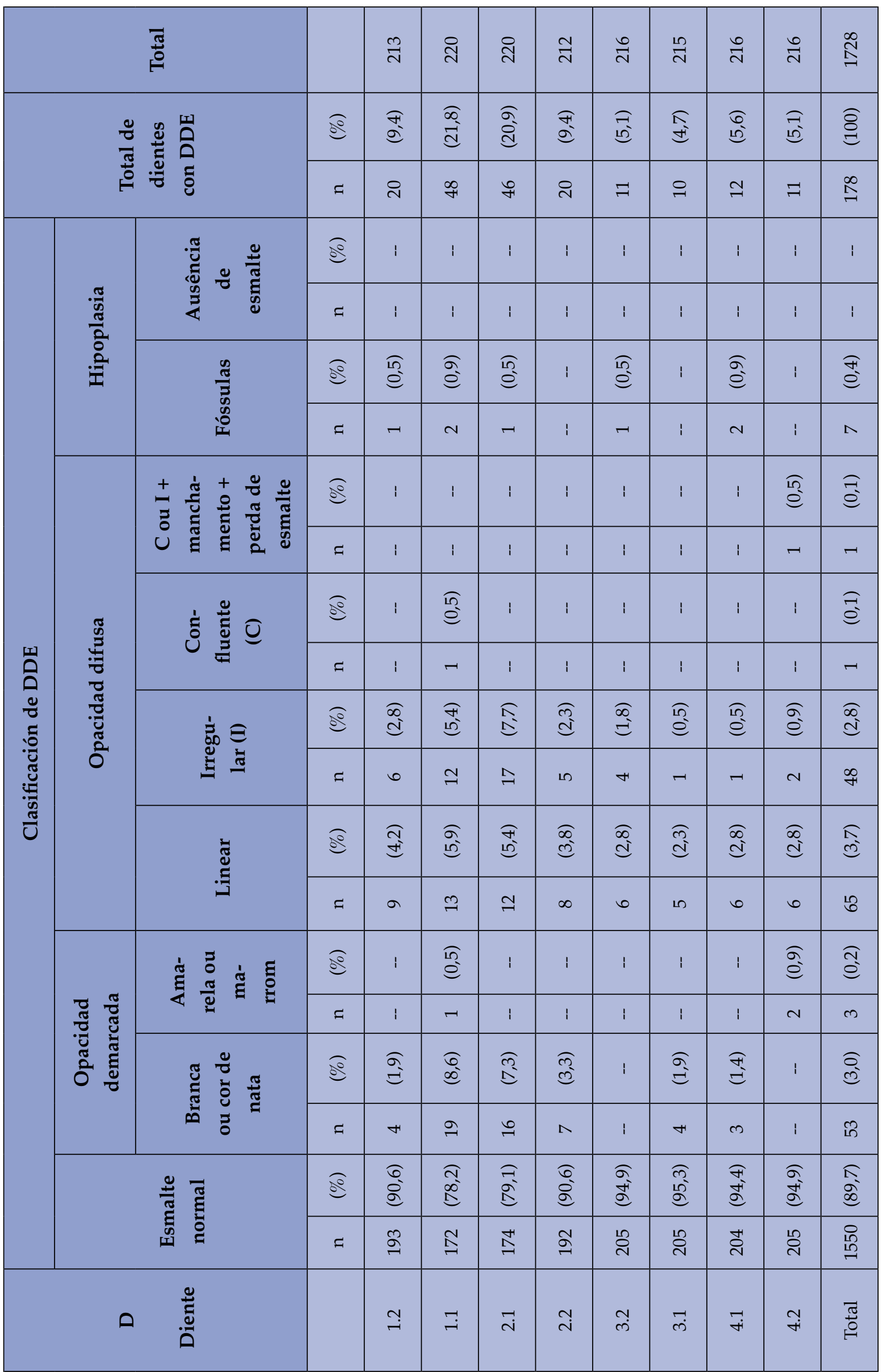


cado con DDE (tabla 2). Los defectos más frecuentemente observados fueron opacidad difusa linear $(3,7 \%)$, opacidad delimitada blanca o crema $(3,0 \% 0$ y opacidad difusa irregular $(2,8 \%)$. A su vez, las alteraciones hipoplásicas fueron las menos prevalentes. Los dientes más afectados fueron los incisivos centrales superiores derechos $(21,8 \%)$ y los menos afectados fueron los incisivos centrales inferiores derechos $(4,7 \%)$.

Los incisivos centrales fueron estadísticamente más afectados por DDE que los incisivos laterales ( $<00,001$; Tabla 3). La prevalencia de estas alteraciones fue 1,84 veces mayor en este grupo de dientes. Considerando apenas los dientes superiores, la razón de prevalencias entre incisivos centrales y laterales sube para 2,27. Con relación a la localización de los dientes, los incisivos superiores presentan una prevalencia de DDE 3,04 veces mayor que los incisivos inferiores, $y$ esta diferencia fue estadísticamente significante $(\mathrm{p}<0,001)$. La posición del diente no alteró de manera significante la prevalencia de DDE ( $\mathrm{p}=0,764$; Tabla 3).

La tabla 4 evidencia la presencia de asociación estadísticamente significante entre el grupo de dientes y el tipo de DDE $(p=0,031)$. Aunque la opacidad difusa haya sido la alteración más frecuente en los dos grupos de dientes, la prevalencia de opacidad demarcada fue mayor en los incisivos centrales (37\%) que los incisivos laterales $(18,3 \%)$, mientras que la prevalencia de opacidades difusas fue mayor en los incisivos laterales $(78,3 \%)$ que entre los incisivos centrales $(58,6 \%)$. La prevalencia de hipoplasia fue similar en am-

Tabla 3. Distribución de los dientes incisivos de los niños escolares de las esculelas públicas de la ciudad de Camboriú, SC, en función de la prevalencia de defectos de desarrollo del esmalte y demás variables independientes. $2002, n=1728$.

\begin{tabular}{|c|c|c|c|c|c|c|c|c|}
\hline \multirow{3}{*}{$\begin{array}{c}\text { Variábles independentes } \\
\text { Grupo de dentes }\end{array}$} & \multicolumn{6}{|c|}{ Defectos de Desarrollo del Esmalte } & \multirow{3}{*}{$\begin{array}{c}\text { valor de } \\
\mathbf{p}^{*}\end{array}$} & \multirow{3}{*}{$\frac{\mathrm{RP}}{\left[\mathrm{IC}_{95 \%}\right]}$} \\
\hline & \multicolumn{2}{|c|}{ Presente } & \multicolumn{2}{|c|}{ Ausente } & \multicolumn{2}{|c|}{ Total } & & \\
\hline & $\mathrm{n}$ & $(\%)$ & $\mathrm{n}$ & $(\%)$ & $\mathrm{n}$ & $(\%)$ & & \\
\hline Incisivos centrales & 116 & $(13,3)$ & 755 & $(86,7)$ & 871 & $(100)$ & \multirow{2}{*}{$\mathrm{p}<0,001$} & $1,84 \#$ \\
\hline Incisivos laterales & 62 & $(7,2)$ & 795 & $(92,8)$ & 857 & $(100)$ & & {$[1,37-2,47]$} \\
\hline \multicolumn{9}{|l|}{ Arcada } \\
\hline Superior & 134 & $(15,5)$ & 731 & $(84,5)$ & 865 & $(100)$ & \multirow{2}{*}{$\mathrm{p}<0,001$} & 3,04 \\
\hline Inferior & 44 & $(5,1)$ & 819 & $(94,9)$ & 863 & $(100)$ & & {$[2,19-4,21]$} \\
\hline \multicolumn{9}{|l|}{ Lado } \\
\hline Derecho & 91 & $(10,5)$ & 774 & $(89,5)$ & 865 & $(100)$ & \multirow{2}{*}{$\mathrm{p}=0,764$} & \multirow{2}{*}{-} \\
\hline Izquierdo & 87 & $(10,1)$ & 776 & $(89,9)$ & 863 & (100) & & \\
\hline
\end{tabular}

*Test Chi-quadrado. RP: Razón de prevalência. [IC95\%]: Intervalo de confiança de 95\%. \#Si considerados apenas los incisivos superiores, os valores de RP e IC pasan a ser 2,27 [1,61-3,21]. 
Tabla 4. Distribución de los dientes incisivos de los niños escolares de la red pública de la ciudad de Camboriú, SC, en función de la clasificación del defecto de desarrollo del esmalte presentado y demás variables independientes. 2002, $n=176 \#$.

\begin{tabular}{|c|c|c|c|c|c|c|c|}
\hline \multirow{3}{*}{$\begin{array}{c}\text { Variables independientes } \\
\text { Grupo de dientes } \\
\end{array}$} & \multicolumn{6}{|c|}{ Defectos de Desarrollo del Esmalte } & \multirow{3}{*}{ valor de $\mathrm{p}^{*}$} \\
\hline & \multicolumn{2}{|c|}{ Opacidad demarcada } & \multicolumn{2}{|c|}{ Opacidad difusa } & \multicolumn{2}{|c|}{ Hipoplasia } & \\
\hline & $\mathrm{n}$ & $(\%)$ & $\mathrm{n}$ & $(\%)$ & $\mathrm{n}$ & $(\%)$ & \\
\hline Incisivos centrales & 43 & $(37,1)$ & 68 & $(58,6)$ & 5 & $(4,3)$ & \multirow{2}{*}{$\mathrm{p}=0,031$} \\
\hline Incisivos laterales & 11 & $(18,3)$ & 47 & $(78,3)$ & 2 & $(3,3)$ & \\
\hline \multicolumn{8}{|l|}{ Arcada } \\
\hline Superior & 47 & $(35,1)$ & 83 & $(61,9)$ & 4 & $(3,0)$ & \multirow{2}{*}{$\mathrm{p}=0,052$} \\
\hline Inferior & 7 & $(16,7)$ & 32 & $(76,2)$ & 3 & $(7,1)$ & \\
\hline \multicolumn{8}{|l|}{ Lado } \\
\hline Derecho & 27 & $(30,3)$ & 57 & $(64,0)$ & 5 & $(5,6)$ & \multirow{2}{*}{$\mathrm{p}=0,529$} \\
\hline Izquierdo & 27 & $(31,0)$ & 58 & $(66,7)$ & 2 & $(2,3)$ & \\
\hline
\end{tabular}

\#Fuerom incluídos en estas análises apenas los dientes que presentavam defecito de desarrollo del esmalte. *Teste Chi-quadrado. Valores de "p" en negrito expresan diferencia estatisticamente significante.

Tabla 5. Distribución de los dientes incisivos de los niños escolares de la red pública de la ciudad de Camboriú, SC, e função de la extensión del defecto de desarrollo del esmalte presentado y demás variables independientes. 2002, $n=176 \#$.

\begin{tabular}{|c|c|c|c|c|c|c|c|}
\hline \multirow{3}{*}{$\begin{array}{l}\text { Variables independentes } \\
\text { Grupo de dientes }\end{array}$} & \multicolumn{6}{|c|}{ Extensión del Defecto de Desarrollo del Esmalte (DDE) } & \multirow{3}{*}{$\begin{array}{l}\text { valor } \\
\text { de } p^{*}\end{array}$} \\
\hline & \multicolumn{2}{|c|}{$\begin{array}{c}\mathrm{DDE} \leq 1 / 3 \mathrm{da} \\
\text { superfície vestibular } \\
\text { coronária }\end{array}$} & \multicolumn{2}{|c|}{$\begin{array}{c}1 / 3<\mathrm{DDE} \leq 2 / 3 \mathrm{da} \\
\text { superfície vestibular } \\
\text { coronária }\end{array}$} & \multicolumn{2}{|c|}{$\begin{array}{l}\mathrm{DDE}>2 / 3 \text { da su- } \\
\text { perfície vestibular } \\
\text { coronária }\end{array}$} & \\
\hline & $\mathrm{n}$ & $(\%)$ & $\mathrm{n}$ & $(\%)$ & $\mathrm{n}$ & $(\%)$ & \\
\hline Incisivos centrales & 80 & $(69,0)$ & 20 & $(17,2)$ & 16 & $(13,8)$ & \multirow{2}{*}{$\mathrm{p}=0,651$} \\
\hline Incisivos laterales & 43 & $(69,3)$ & 13 & $(21,0)$ & 6 & $(9,7)$ & \\
\hline \multicolumn{8}{|l|}{ Arcada } \\
\hline Superior & 87 & $(64,9)$ & 26 & $(19,4)$ & 21 & $(15,7)$ & \multirow{2}{*}{$\mathrm{p}=0,041$} \\
\hline Inferior & 36 & $(81,8)$ & 7 & $(15,9)$ & 1 & $(2,3)$ & \\
\hline \multicolumn{8}{|l|}{ Lado } \\
\hline Derecho & 64 & $(70,3)$ & 16 & $(17,6)$ & 11 & $(12,1)$ & \multirow{2}{*}{$\mathrm{p}=0,931$} \\
\hline Izquierdo & 59 & $(67,8)$ & 17 & $(19,5)$ & 11 & $(12,6)$ & \\
\hline
\end{tabular}

\#Fuerom incluídos en estas análises apenas los dentes que presentaban defecto de desarrollo del esmalte. *Test Chi-quadrado. Valores de " $\mathrm{p}$ " en negrito expressan diferencia estatisticamente significante. 
bos grupos de dientes. El tipo de defecto de esmalte no fue influenciado por la localización del diente (Tabla 4).

A su vez, el grado en que los dientes se vieron afectados por DDE fue similar entre los incisivos centrales y los incisivos laterales y entre derecha e izquierda (Tabla 5). Sin embargo, los dientes superiores tenían una mayor extensión de la corona afectados por defectos en el desarrollo que los dientes inferiores. Mientras que el 15,7\% de los incisivos superiores afectados presentaban más de $2 / 3$ de la superficie vestibular de la corona afectado por el DDE, sólo 2,3\% de los incisivos inferiores mostró DDE con la misma extensión, y esta diferencia fue estadísticamente significativa ( $p=0,041$; Tabla 5).

\section{Discusión}

El estudio de los problemas de desarrollo del esmalte (DDE) es de gran importancia debido al aumento de la susceptibilidad en el desarrollo de lesiones de carie (Hoffmann, de Souza y Cypriano, 2007), la posible interferencia con la estética (Ellwood y O'Mullane, 1995a) y de la imagen que el individuo hace de sí mismo ( $\mathrm{Su}$ jak, Kadir Sol y de 2004, Marshman, Gibson, Robinson, 2009). Además, son buenos indicadores de la calidad de vida de una población dada, ya que están íntimamente asociados a alteraciones sistémicas, el resultado del patrón de salud y, en consecuencia de las condiciones socioeconómicas.

Considerando su importancia, varios estudios se han llevado a cabo, y, en gran parte los métodos de examen son exclusivamente clínicos. En este estudio se optó por utilizar un registro fotográfico de los incisivos permanentes para el diagnóstico de DDE. Este abordaje fue adoptado por razones prácticas, ya que requiere muy poco tiempo con el niño y, en consecuencia, en la institución educativa, lo que permitió la realización del estudio sin interferencia en las actividades escolares. Además, para los estudios de DDE en la región anterior, el método fotográfico ha mostrado una buena concordancia con el método clínico (Ellwood, Cortés, O'Mullane, 1996; Rugg-Goma, Al Mohamadi, Butler, 1997, Wong et al, 2005). En relación al examen exclusivo de los incisivos, aunque esta estrategia no es recomendable para la evaluación de DDE en la dentición decidua, que demuestra una mayor participación de los dientes posteriores (Lunardelli y Peres, 2005), en la dentición permanente se justifica porque son los dientes incisivos más afectados (Dummer et al 1986 b, 1986, Dummer et al, Rugg-Goma, Mohamadi Al, Butler, 1997). Por lo tanto, se puede considerar que la evaluación de dientes anteriores permanentes es un fuerte indicativo de la manifestación de DDE en una determinada población.

Para analizar los datos deben considerarse que los DDE presentan gran sensibilidad a las condiciones de vida de cada población (Rugg-Gum, Al Mohamadi, Butler, 1997), la comparación entre los estudios epidemiológicos debe ser cuidadosa pues existen diferencias entre las poblaciones.

En este estudio, realizado en poblaciones que reciben agua fluorada, se observa una alta prevalencia de DDE considerando al individuo como unidad (34,8\% del total de los niños) o la unidad diente (10,3\% del total de dientes), tablas $\mathbf{1}$ y 2 . Parte de los resultados pueden ser explicados ya que el índice utilizado incluye las alteraciones producidas en el esmalte por el uso de fluoruros. Las comunidades que reciben agua fluorada tienen una mayor prevalencia de DDE, cuando son evaluadas por medio de este índice (RuggGoma, Al Mohamadi, Butler, 1997; Kanagarat- 
nam et al, 2009). Sin embargo, Machiulskiene et al. en 2009, demostró que si se excluyen las lesiones con las características de fluorosis del índice de DDE, la prevalencia de las alteraciones del esmalte disminuyen en presencia del agua fluorada.

En este estudio, encontramos que el defecto mas prevalerte fue la opacidad difusa linear, seguido de la opacidad demarcada blanco o crema y la opacidad difusa irregular (Tabla 2). A pesar de que la opacidad difusa haya sido la alteración más frecuente, la prevalencia de opacidad demarcada fue mayor entre los incisivos centrales que en los incisivos laterales (Tabla 3). Este aspecto puede explicarse por la asociación entre la opacidad demarcada y factores locales, tales como trauma o lesiones periapicales en dientes deciduos, aspectos para los que los incisivos centrales son los más susceptibles.

Los estudios indican que la opacidad difusa está directamente relacionada con el consumo de flúor (Rugg-Gunn, Al Mohamadi, Butler, 1997) siendo el defecto más comúnmente encontrado en poblaciones con suministro de agua fluorada, mientras que, en regiones sin o con bajos niveles de fluoruro en el agua potable, el defecto más común es la opacidad delimitada (Dini et al, 2000, Ellwood, O'Mullane, 1995).

La ausencia de diferencia estadísticamente significante para prevalencia de los defectos de esmalte en relación al género (tabla 1) corrobora con diversos estudios (Hoffman et al 1988, King 1989, Crooks, 1990, Hoffmann, Sousa; Cypriano, 2007) donde esta variable no estaba asociada a la prevalencia de DDE. Debido a la ausencia de diferencias significativas entre los géneros, por lo que fue sugerido que en la interpretación de los datos sea considerada la muestra final.
La literatura ha demostrado que los dientes superiores son mas afectados que los inferiores (Dooland e Wyllie, 1989) en especial los incisivos centrales. La mayor prevalencia de DDE en los dientes superiores y en incisivos centrales (tabla 4) puede ser justificada debido a la alta frecuencia de traumas en los incisivos centrales deciduos (Mestrinho; Bezerra; Carvalho, 1998), siendo que Andreasen; Sundströn y Ravn (1971) afirman que es de fácil comprensión que injurias en la dentición decidua afecten directamente a la dentición permanente por íntima relación entre los ápices de los dientes deciduos y el germen de los permanentes. En un amplio estudio, Da Silva et al, 2009, evaluando 623 dientes permanentes de niños que sufrieron traumas en dientes deciduos, observó que los defectos de desarrollo ocurrieron en $20,2 \%$ de los dientes permanentes, siendo que la opacidad de esmalte blanca o marrón-amarilla fue el disturbio más prevalente.

Con relación a la extensión de las lesiones, la gran mayoría presentó alteraciones que no sobrepasan $1 / 3$ de la superficie vestibular, resultando que en los incisivos superiores se encontró lesiones con mayor extensión, probablemente por ser los más expuestos a los traumatismos (tabla 5). Resultados semejantes fueron encontrados por Hoffmann, Cutress e Tomiki, 1988; Nunn et al. (1993) e Yusoff et al. (2008).

Los resultados de este trabajo permiten que estudios futuros puedan monitorear las alteraciones del esmalte de esta comunidad, las cuales presentan especial importancia ya que estas entidades clínicas pueden ser reflejo tanto de mudanzas de cualidad de vida (factores nutricionales, violencia, traumatismos) como alteraciones en el acceso al flúor. 


\section{Conclusiones}

La prevalencia de DDE en la población estudiada fue alta, con preponderancia de las opacidades difusas. Los incisivos centrales superiores fueron afectados con más frecuenciasa y mayor gravedad. Este conocimiento sobre la prevalencia y distribución de los diferentes tipos de DDE contribuirán para mejorar el monitoreo de las condiciones de salud de la población.

\section{Bibliografia}

1. Andreasen JO, Sundstrom B, Ravn JJ. The effect of traumatic injuries to primary teeth on their permanent successors. A clinical and histologic study of 117 injured permanent teeth. Scand J Dent Res 1971; 79(4):219-83.

2. Arnadóttir IB, Sigurións H, Holbrook WP. Enamel opacities in 8-year-old Icelandic children in relation to their medical history as infants. Community Dent Health 2005;22(4):279-81.

3. Assunção CM, Falleiros T, Gugisch RC, Fraiz FC, Losso EM. Tetralogia de Fallot e sua repercussão na saúde bucal. Rev Paul Pediatr 2008;26(1):93-6.

4. Cordeiro MM, Rocha MJ. The effects of periradicular inflamation and infection on a primary tooth and permanent successor. J Clin Pediatr Dent 2005;29(3):193-200.

5. Commission on Oral Health, Research and Epidemiology. FDI. A review of developmental defects of enamel index (DDE index). International Dental Journal 1992;42(6):411-26.

6. Crooks MC. Prevalente of developmental defects of enamel in children and young adults in the Cook Islands. N Z Dent J 1990;86(384):39-41.

7. Dini EL, Holt RD, Bedi R. Prevalence of caries and developmental defects of enamel in 9-10 year old children living in areas in Brazil with differing water fluoride histories. Brit Dent J 2000;188(3):146-9.

8. Dooland MB, Wylie A. A photographic study of enamel defects among South Australian school children. Australian Dental Journal 1989;34(5):470-3.

9. Dummer PM, Kingdon A, Kingdon R. Prevalence of enamel defects in a group of 11-and 12-year-old chidren in South Wales. Community Dent Oral Epidemiol 1986;14(2):119-22.

10. Dummer PM, Kingdon A, Kingdon R. Distribution of developmental defects of tooth enamel by tooth-type in 11-12-year-old chidren in South Wales. Community Dent Oral Epidemiol 1986;14(6):341-4.

11. Ellwood RP, O’Mullane DM. Enamel opacities and dental esthetics. J Public Health Dent 1995a;55(3):171-6

12. Ellwood RP, O'Mullane DM. Dental enamel opacities in Three Groups with Varying Levels of Fluoride in Their Drinking Water. Caries Res 1995b;29(2):137-42.

13. Ellwood RP, Cortea DF, O'Mullane DM. A photographic study of developmental defects of enamel in Brazilian school chidren. Int Dent J 1996;46(2):69-75.

14. do Espírito Santo Jácomo DR, Campos V. Prevalence of sequelae in the permanent anterior teeth after trauma in their predecessors: a longitudinal study of 8 years. Dent Traumatol 2009;25(3):300-4.

15. Guergolette RP, Dezan CC, Frossard WTG, Ferreira FBA, Cerci Neto A, Fernandes KBP. Prevalence of developmental defects of enamel in children and adolescents with asthma. J Bras Pneumol 2009;35(4):295-300.

16. Gonzales-Pinedo CO, Perona-Miguel de Priego G. Amelogenésis imperfecta: Criterios de clasificación y aspectos genéticos. Rev Estomatol Herediana 2009;19(1):55-62.

17. Griffin RC, Donlon D. Patterns in dental enamel hypoplasia by sex and age at death in two archaeological populations. Arch Oral Biol 2009;54(1):93-100.

18. Hoffman MP, Cutress TW, Tomiki S. Prevalence of developmental defects of enamel in children in the Kingdom of Tonga. New Zealand Dent J 1988;84(375):7-10. 
19. Hoffmann RHS, Sousa MLR, Cypriano S. Prevalência de defeitos de esmalte e sua relação com cárie dentária nas dentições decídua e permanente, Indaiatuba, São Paulo, Brasil. Cad. Saúde Pública 2007;23(2):435-44.

20. Kanagaratnam S, Schluter P, Durward C, Mahood R, Mackay T. Enamel defects and dental caries in 9-year-old children living in fluoridated and nonfluoridated areas of Auckland, New Zealand. Community Dent Oral Epidemiol 2009;37(3):250-9.

21. King NM, Wei SH. Developmental defects of enamel: A study of 12-years-old in Hong Kong. JADA 1986;112(6):835-9.

22. King NM. Developmental Defects of Enamel In Chinese Girls And Boys In Hong Kong. Adv Dent Res 1989;3(2):120-5.

23. Lunardelli SE, Peres MA. Prevalence and distribution of developmental enamel defects in the primary dentition of pre-school children. Braz Oral Res 2005;19(2):144-9.

24. Marshman Z, Gibson B, Robinson PG. The impact of developmental defects of enamel on young people in the UK. Community Dent Oral Epidemiol 2009;37(1):45-57.

25. Massoni ACLT, Oliveira AFB, Chaves AMB, Sampaio FC, Rosenblatt A. Fatores sócio-econômicos relacionados ao risco nutricional e sua associação com a freqüência de defeitos do esmalte em crianças da cidade de João Pessoa, Paraíba, Brasil. Cad. Saúde Pública 2007;23(12):2928-37.

26. Massoni AC, Chaves AM, Rosenblatt A, Sampaio FC, Oliveira AF. Prevalence of enamel defects related to pre-, periand postnatal factors in a Brazilian population. Community Dent Health 2009;26(3):143-9.

27. Majorana A, Bardellini E, Ravelli A, Plebani A, Polimeni A, Campus G. Implications of gluten exposure period, CD clinical forms, and HLA typing in the association between celiac disease and dental enamel defects in children. A casecontrol study. Int J Paediatr Dent 2010;20(2):119-24.

28. Mestrinho HD, Bezerra ACB, Carvalho JC. Traumatic Dental Injuries in Brazilian Pre-school children. Braz Dent J 1998;9(2):101-4.

29. Nunn JH, Ekanavake L, Rugg-Gunn AJ, Saparamadu KD. Assessment of enamel pacities in children in Sri Lanka and England using a photographic method. Community Dent Health 1993;10(2):175-88.

30. da Silva Assunção LR, Ferelle A, Iwakura ML, Cunha RF. Effects on permanent teeth after luxation injuries to the primary predecessors: a study in children assisted at an emergency service. Dent Traumatol 2009;25(2):165-70.

31. Rugg-Gunn AJ, al-Mohammadi SM, Butler TJ. Effects of fluoride level in drinking water, nutritional status, and socioeconomic status on the prevalence of developmental defects of dental enamel in permanent teeth in Saudi 14-year-old boys. Caries Res 1997;31(4):259-67.

32. Sujak SL, Kadir RA, Dom TNM. Esthetic perception and psychosocial impact of developmental enamel defects among Malaysian adolescents. Journal of Oral Science 2004;46(4):221-6.

33. Wong HM, McGrath C, Lo ECM, King NM. Photographs as a means of assessing developmental defects of enamel. Community Dent Oral Epidemiol 2005;33(6):438-46.

34. Yusoff N, Jaafar N, Razak IA, Chew YY, Ismail N, Bulgiba AM. The prevalence of enamel opacities in permanent teeth of 11-12 year-old school children in Kuala Lumpur, Malaysia. Community Dent Health 2008;25(1):55-8.

Recibido: 08-07-2010

Aceptado: 22 -09- 2010

Correspondencia: fraiz@ufpr.br 